\title{
Developmental modification of lipophosphoglycan during the differentiation of Leishmania major promastigotes to an infectious stage
}

\author{
Malcolm J.McConville ${ }^{1}$, Salvatore J.Turco², \\ Michael A.J.Ferguson ${ }^{1}$ and David L.Sacks ${ }^{3}$ \\ 'Department of Biochemistry, University of Dundee, Dundee DD1 \\ $4 \mathrm{HN}$, UK, ${ }^{2}$ Department of Biochemistry, University of Kentucky \\ Medical Center, Lexington, KY and ${ }^{3}$ Laboratory of Parasitic Diseases, \\ NIAID, NIH, Bethesda, MD, USA \\ Communicated by P.Cohen (Dundee)
}

Protozoan parasites of the genus Leishmania produce the novel surface glycoconjugate, lipophosphoglycan (LPG), which is required for parasite infectivity. In this study we show that LPG structure is modified during the differentiation of L.major promastigotes from a less infectious form in logarithmic growth phase to a highly infectious 'metacyclic' form during stationary growth phase. In both stages, the LPGs comprise linear chains of phosphorylated oligosaccharide repeat units which are anchored to the membrane via a glycosylphosphatidylinositol glycolipid anchor. During metacyclogenesis there is (i) an approximate doubling in the average number of repeat units per molecule from 14 to 30 , (ii) a pronounced decrease in the relative abundance of repeat units with side chains of $\beta \mathrm{Gal}$ or $\mathrm{Gal} \beta 1-3 \mathrm{Gal} \beta 1$-, and a corresponding increase in repeat units with either no side chains or with side chains of $\operatorname{Arap} \alpha 1-2 \mathrm{Gal} \beta 1$ and (iii) a decrease in the frequency with which the glycolipid anchor is substituted with a single glucose$\alpha 1$-phosphate residue. While the majority of the LPG phosphoglycan chains are capped with the neutral disaccharide, Man $\alpha 1-2 \mathrm{Man}$, a significant minority of the chains appeared to terminate in non-phosphorylated repeat units and may represent incompletely capped species. We suggest that the developmental modification of LPG may be important in modulating the binding of promastigotes to receptors in the sandfly midgut and on human macrophages and in increasing the resistance of metacyclic promastigotes to complement-mediated lysis. Key words: differentiation/glycosylated phosphatidylinositol/ Leishmania/lipophosphoglycan

\section{Introduction}

Protozoan parasites of the genus Leishmania cause a number of important human diseases, including cutaneous, visceral and mucocutaneous leishmaniasis. During their digenetic life cycle, these parasites alternate between an extracellular promastigote stage in the digestive tract of the sandfly vector and an intracellular amastigote stage that resides within the phagolysosome compartment of macrophages in the mammalian host. One of the key determinants of parasite infectivity and survival in these environments is the surface glycoconjugate, lipophosphoglycan (LPG) (reviewed by Turco, 1989; McConville, 1991). LPG is produced by all
Leishmania species and is the major macromolecule on the surface of the promastigote stage. Within the mammalian host it is thought to play a role in complement activation and resistance to complement-mediated lysis (Puentes $e t$ al., 1988, 1990), in the attachment and entry of promastigotes into mammalian macrophages (Handman and Goding, 1985; Talamas-Rohana et al., 1990; da Silva et al., 1989) and in survival in the phagolysosomal compartment (McNeely and Turco, 1990; Elhay et al., 1990).

Recent studies on the LPGs from three species of Leishmania indicate that the backbone structure of these molecules is highly conserved. They all consist of polymers of the phosphorylated disaccharide, $\mathrm{PO}_{4}-6 \mathrm{Gal} \beta 1-4 \mathrm{Man} \alpha 1-$ which are anchored to the plasma membrane via a novel glycosyl-phosphatidylinositol glycolipid anchor (Turco et al., 1989; McConville et al., 1990a; Ilg et al., 1992; Thomas et al., 1992). Interspecies polymorphisms occur in the nature and frequency of oligosaccharide side chains that branch from the galactose residues in the repeat unit backbone. Thus in the LPG from L.donovani, none of the repeat units are substituted with side chains (Turco et al., 1987), while in the L.mexicana and L.major LPGs, the repeat units are variably substituted either with single glucose residues (Ilg et al., 1992) or with monosaccharide or oligosaccharide side chains of galactose and arabinose residues (McConville et al., 1990a), respectively. Additional interspecies polymorphism in LPG structure may also occur in the nature of the predominant neutral, mannose-containing oligosaccharides that cap the non-reducing terminus of the phosphoglycan chains (McConville et al., 1990a; Ilg et al., 1992; Thomas et al., 1992).

It is now clear that promastigotes undergo morphological and biochemical changes within the midgut of the sandfly vector which lead to an increase in their infectivity in the mammalian host (Sacks and Perkins, 1984; Davies et al., 1990). This process can be mimicked in vitro when promastigotes develop from a less infective form during logarithmic growth to a highly infective form during stationary phase (reviewed in Sacks, 1989). We propose to use the terms procyclic and metacyclic to refer to these respective promastigote developmental stages. L.major metacyclic promastigotes can be distinguished from procyclic promastigotes by their failure to be agglutinated by low concentrations of the lectin peanut agglutinin and by their reaction with stage-specific monoclonal antibodies (Sacks and da Silva, 1987). In the case of L.major, these reagents recognize surface LPG, raising the possibility that stagespecific polymorphisms in LPG structure may play a role in determining parasite virulence. Preliminary studies have indicated that there is both an increase in the molecular weight of LPG and a change in the relative abundance of the major repeat units during metacyclogenesis (Sacks et al., 1990). In this study, we have further analyzed the LPGs from procyclic and metacyclic promastigotes to characterize fully the nature of these changes. The results have implications 


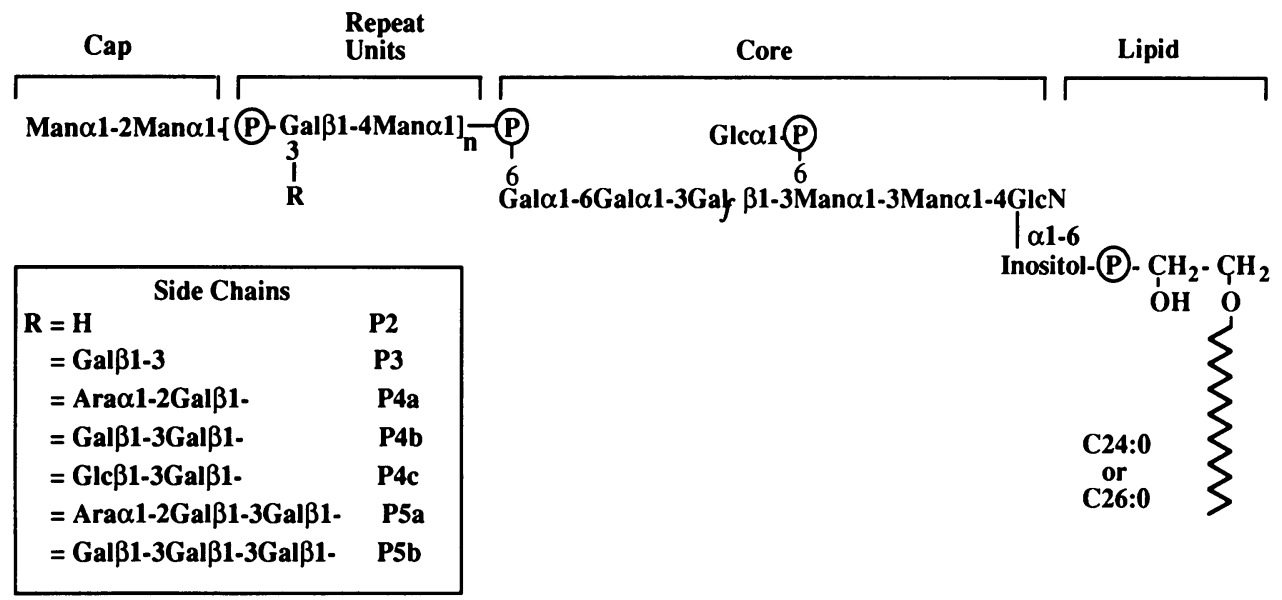

Fig. 1. Summary of the structure of L.major LPG (from McConville et al., 1987, 1990b and McConville and Homans, 1992). The number of repeat units per phosphoglycan chain (n) varies from 5 to 40 . All residues are in the pyranose configuration unless otherwise noted.

for understanding how LPG mediates binding of promastigotes to the sandfly midgut and macrophages of the mammalian host, and its role in increasing the resistance of metacyclic promastigotes to complement-mediated lysis.

\section{Results}

Procyclic and metacyclic promastigotes were obtained from in vitro cultures of $L$.major promastigotes in logarithmic and late stationary growth phases, respectively. In the case of the metacyclic promastigotes, a relatively homogeneous population was obtained after removal of remaining procyclic forms by agglutination with peanut agglutinin $(50 \mu \mathrm{g} / \mathrm{ml})$. LPG from procyclic and metacyclic promastigotes (P-LPG and M-LPG, respectively) was extracted from the delipidated cells in 9\% 1-butanol and purified by octyl-Sepharose chromatography for structural analysis. We have shown previously that L.major LPG consists predominantly of repeating phosphorylated oligosaccharide units, all of which contain the backbone structure, $\mathrm{PO}_{4}-6 \mathrm{Gal} \beta 1-4 \mathrm{Man}$, where the 3-position of the Gal residue may either be unsubstituted (i.e. P2) or substituted with side chains of $\beta 1-3$ linked Gal residues (i.e. P3, P4b, P5b) some of which may terminate in $\alpha \mathrm{Ara}_{p}(\mathrm{P} 4 \mathrm{a}, \mathrm{P} 5 \mathrm{a})$ or $\beta \mathrm{Glc}(\mathrm{P} 4 \mathrm{c})$ residues (McConville et al., 1990a, see Figure 1). The relative abundance of these different repeat units in P-LPG and M-LPG was determined by Dionex HPLC of the phosphorylated oligosaccharides after mild acid depolymerization of the LPGs and reduction of the released oligosaccharides with $\mathrm{NaB}_{3} \mathrm{H}_{4}$. The structures of the labelled repeat units (Table I) were confirmed by (i) their comigration with unlabelled authentic standards, (ii) methylation analysis of the dephosphorylated repeat units and (iii) microsequencing of the dephosphorylated repeat units with $40 \mathrm{mM}$ trifluoroacetic acid $(60 \mathrm{~min}$, $100^{\circ} \mathrm{C}$ ) to cleave $\alpha$ Ara residues and with bovine testicular $\beta$-galactosidase to remove terminal $\beta \mathrm{Gal}$ residues as described previously (results not shown, McConville $e t$ al. 1990a). Comparison of the profiles from P-LPG and M-LPG show that there are marked quantitative differences in the relative amounts of the major repeat units (Figure 2, Table I). In P-LPG, $\sim 80 \%$ of the repeat units were substituted with side chains of $\mathrm{Gal} \beta 1-3, \mathrm{Gal} \beta 1-3 \mathrm{Gal} \beta 1-3$ or $\mathrm{Gal} \beta 1-3 \mathrm{Gal} \beta 1-3 \mathrm{Gal} \beta 1-3$. Only a minority $(11 \%)$ of the repeat units were substituted with side chains that terminated in $\alpha$-arabinopyranose residues. In contrast, only a minority 3594
(39\%) of the repeat units in M-LPG contained $\beta$ Galterminating side chains, while most of the repeat units were either unsubstituted or substituted with side chains of Arap $\alpha 1-2 \mathrm{Gal} \beta 1$ - (Table I).

Previous analyses have indicated that there is an increase in the molecular weight of the LPG during metacyclogenesis (Sacks et al., 1990). This was confirmed by determination of the ratio of mannose to 1-O-alkylglycerol in each LPG preparation, which indicated that the average number of repeat units per molecule increased from 14 to 30 during metacyclogenesis (Table I). These analyses also showed that the predominant alkyl chains in the lyso-PI anchor were C24:0 (73\%) and C26:0 (21\%), respectively (results not shown). Gel permeation HPLC of the galactose oxidase $/ \mathrm{NaB}_{3} \mathrm{H}_{4}$-labelled phosphoglycan moieties revealed that both LPG preparations were polydisperse with regard to their molecular weight (Figure 3A and B). The elution positions of phosphoglycan moieties with different degrees of polymerization were calculated from the ratio of mannose to myo-inositol in fractions across the elution profile. These results indicate that most of the phosphoglycan chains of $\mathrm{P}$ LPG have between eight and 30 repeat units. While some of the phosphoglycan chains of M-LPG eluted in this region, most of these chains eluted at or near the void volume. Determination of the mannose to myo-inositol ratio in the voided material indicated that the phosphoglycan chains in this fraction had, on average, 35 repeat units per molecule (Figure 3B). M-LPG was also polydisperse with regard to the relative abundance of the repeat units with arabinosylated side chains. In particular, monosaccharide analysis of the size fractionated material indicated that the longer phosphoglycan chains were enriched in repeat units with the Ara-terminating side chains (Figure 3C). This was confirmed by analysis of the high molecular weight phosphoglycan chains of M-LPG which eluted in the void volume of the gel permeation column. These chains were enriched for P4a and $\mathrm{P} 2$, but still contained a significant number $(21 \%)$ of repeat units with $\beta \mathrm{Gal}$-terminating side chains (Table I).

Differences were also observed in the glycan cores of Pand M-LPG. The glycan cores were obtained by nitrous acid deamination and $\mathrm{NaB}_{3} \mathrm{H}_{4}$ reduction of the acid-released GPI anchors. Dionex HPLC of the labelled glycan cores revealed that P-LPG only contained the diphosphorylated hexasaccharide core (Figure 4). We have previously shown that the phosphate groups are located on the terminal Gal 
Table I. Structures and relative abundance of the different phosphorylated repeat units in the LPG from procyclic (P-LPG) and metacyclic (M-LPG) promastigotes and in a high molecular weight pool of M-LPG

\begin{tabular}{|c|c|c|c|c|}
\hline \multirow[t]{2}{*}{ Structure } & & \multicolumn{3}{|c|}{ Mole \% } \\
\hline & & P-LPG & M-LPG & High mol. wt M-LPG ${ }^{a}$ \\
\hline P2 & $\mathrm{PO}_{4}-6 \mathrm{Gal} \beta 1-4 \mathrm{Man}$ & 7 & 15 & 19 \\
\hline P3 & $\mathrm{Gal} \beta 1-3\left[\mathrm{PO}_{4}-6\right] \mathrm{Gal} \beta 1-4 \mathrm{Man}$ & 52 & 31 & 21 \\
\hline P4a & $\operatorname{Ara} \alpha 1-2 \mathrm{Gal} \beta 1-3\left[\mathrm{PO}_{4}-6\right]$ Gal $\beta 1-4 \mathrm{Man}$ & 9 & 45 & 54 \\
\hline P4b & $\mathrm{Gal} \beta 1-3 \mathrm{Gal} \beta 1-3\left[\mathrm{PO}_{4}-6\right] \mathrm{Gal} \beta 1-4 \mathrm{Man}$ & 25 & 6 & 3 \\
\hline P4c & $\mathrm{Glc} \beta 1-3 \mathrm{Gal} \beta 1-3\left[\mathrm{PO}_{4}-6\right] \mathrm{Gal} \beta 1-4 \mathrm{Man}$ & 1 & 1 & $\operatorname{tr}$ \\
\hline P5a & $\operatorname{Ara} \alpha 1-2 \mathrm{Gal} \beta 1-3 \mathrm{Gal} \beta 1-3\left[\mathrm{PO}_{4}-6\right] \mathrm{Gal} \beta 1-4 \mathrm{Man}$ & 2 & 3 & 3 \\
\hline P5b & $\mathrm{Gal} \beta 1-3 \mathrm{Gal} \beta 1-3 \mathrm{Gal} \beta 1-3\left[\mathrm{PO}_{4}-6\right] \mathrm{Gal} \beta 1-4 \mathrm{Man}$ & 4 & 2 & 2 \\
\hline \multicolumn{2}{|c|}{ Average number of repeat units/molecule ${ }^{b}$} & 14 & 30 & ND \\
\hline
\end{tabular}

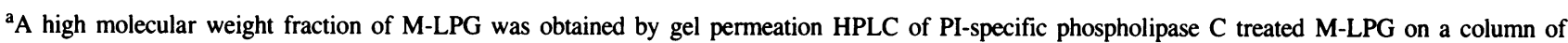
TSK2000SW. Material that eluted in or near the void volume (see Figure 3) was analyzed in the same way as described for the intact LPG fractions. bstimated from the molar ratio of Man/1-O-alkylglycerol, based on the finding that there is one mannose residue per repeat unit and an average of one and two neutral mannose residues in the core and cap structures, respectively.

ND, not determined; tr, trace $(<0.5 \%)$.

residue and on the second Man residue which is distal to the glucosamine of the hexasaccharide core (McConville et al., 1990a). In the intact LPG, these phosphate groups are substituted with the chains of repeat units or with a single $\alpha$ Glc residue, respectively (see Figure 1, McConville and Homans, 1992; Thomas et al., 1992). In contrast, M-LPG contained both diphosphorylated and monophosphorylated cores in approximately equal abundance (Figure 4). The phosphate on the monophosphorylated core was located exclusively on the terminal Gal residue, deduced from the elution position of the labelled core on Dionex HPLC and from the finding that mild acid hydrolysis of the purified labelled species, under conditions which selectively cleave the galactofuranosidic linkage ( $40 \mathrm{mM}$ trifluoroacetic acid, $1 \mathrm{~h}, 100^{\circ} \mathrm{C}$ ), generated a neutral product that comigrated with Man $\alpha 1-3$ Man $\alpha 1-4$ AHM (results not shown). These results indicate that there is a pronounced decrease in the extent to which the glycan core is substituted with the Glc $\alpha 1-\mathrm{PO}_{4}$ moiety in M-LPG.

Analysis of the neutral cap oligosaccharides of P- and M-LPG by Dionex HPLC revealed the presence of a single major species and four minor species in both preparations (Figure 5). The major neutral oligosaccharide coeluted with the previously characterized cap disaccharide, Man $\alpha 1-2 \mathrm{Man}$, and was shown to have this sequence by methylation analysis (Table II). Similarly, three of the minor species were identified, from their coelution with authentic standards and methylation analysis (Table II), as the non-phosphorylated derivatives of P2, P3 and P4a (Figure 5). The fourth minor 'cap' species was shown by methylation analysis (Table II) and $\beta$-galactosidase digestions after mild acid hydrolysis (data not shown) to have the novel sequence Arap1-2Gal/1-4Man. It is likely that all the LPG chains terminate with one of these neutral oligosaccharides, as determination of the ratios of total cap species to total repeat units by Dionex HPLC gave values (1:12 and 1:25 for P-LPG and M-LPG, respectively) that were similar to those expected from the estimates of the average number of repeat units per molecule.

\section{Discussion}

LPG is the major macromolecule on the promastigote surface and is thought to play a key role in parasite survival both in the insect vector and in the mammalian host.
Understanding the ways in which the structures of the LPGs are modified in different developmental stages has provided insights into the structure-function relationships of these molecules and has also suggested important roles for LPG that have not previously been considered. The major changes in LPG structure which occur during metacyclogenesis are summarized in Figure 6. These include an approximate doubling in the average number of repeat units per molecule, from 14 to 30 , and a dramatic increase in the frequency with which the backbone disaccharide repeat units are substituted with short side chains of Ara $\alpha 1-2 \mathrm{Gal} \beta 1-3$. The concomitant decrease in the frequency of side chains with terminal $\beta \mathrm{Gal}$ residues may account for the decreased binding of peanut agglutinin to metacyclic promastigotes, which has become an important criterion for both identifying and purifying this developmental stage. Developmentally regulated differences were also found in the glycan core of the LPGs which was fully substituted with a Glc $\alpha 1-\mathrm{PO}_{4}$ residues in P-LPG but only partially substituted with this residue in M-LPG. In contrast, the nature of the terminal cap domain was relatively constant in both developmental stages. While the majority of the phosphoglycan chains were capped with the disaccharide, Man $\alpha 1-2 M a n$, a significant minority of the chains appeared to terminate in non-phosphorylated repeat units. It is not known whether these molecules represent intracellular precursor species that are still undergoing chain elongation or uncapped molecules that have been transported to the cell surface.

It has been estimated that there are $\sim 3-5 \times 10^{6}$ copies of LPG in the promastigote plasma membrane (McConville and Bacic, 1990), consistent with it being the major macromolecule in the surface glycocalyx that coats the promastigote cell surface (Pimenta et al., 1989). The approximate doubling in the length of the phosphoglycan chains during metacyclogenesis is consistent with the finding that there is a corresponding increase in the thickness of this surface coat (Pimenta et al., 1989). This increase in coat thickness is associated with the appearance of densely packed filamentous structures, which are $\sim 44 \mathrm{~nm}$ long and $5 \mathrm{~nm}$ wide, on the surface of fracture-flipped membranes of metacyclic promastigotes. Molecular modelling of the LPG phosphoglycan backbone (Homans et al., 1992) predicts that many of the M-LPG chains will have similar dimensions (40 
$\mathrm{nm}$ long, $1 \mathrm{~nm}$ wide), raising the possibility that the filamentous structures comprise one or more LPG chains. The absence of similar structures on the surface of procyclic promastigotes (Pimenta et al., 1989) may reflect differences in the surface expression of LPG and/or the inability of the shorter P-LPG chains to form these complexes.

The developmental modification of LPG during metacyclogenesis could occur either as the result of elongation of endogenous P-LPG or by de novo synthesis of M-LPG with concomitant loss (by catabolism or shedding) of P-LPG, or by a combination of both mechanisms. It is not possible to distinguish between these different mechanisms from the structural data. For example, although there is a decrease in the relative abundance of Gal-

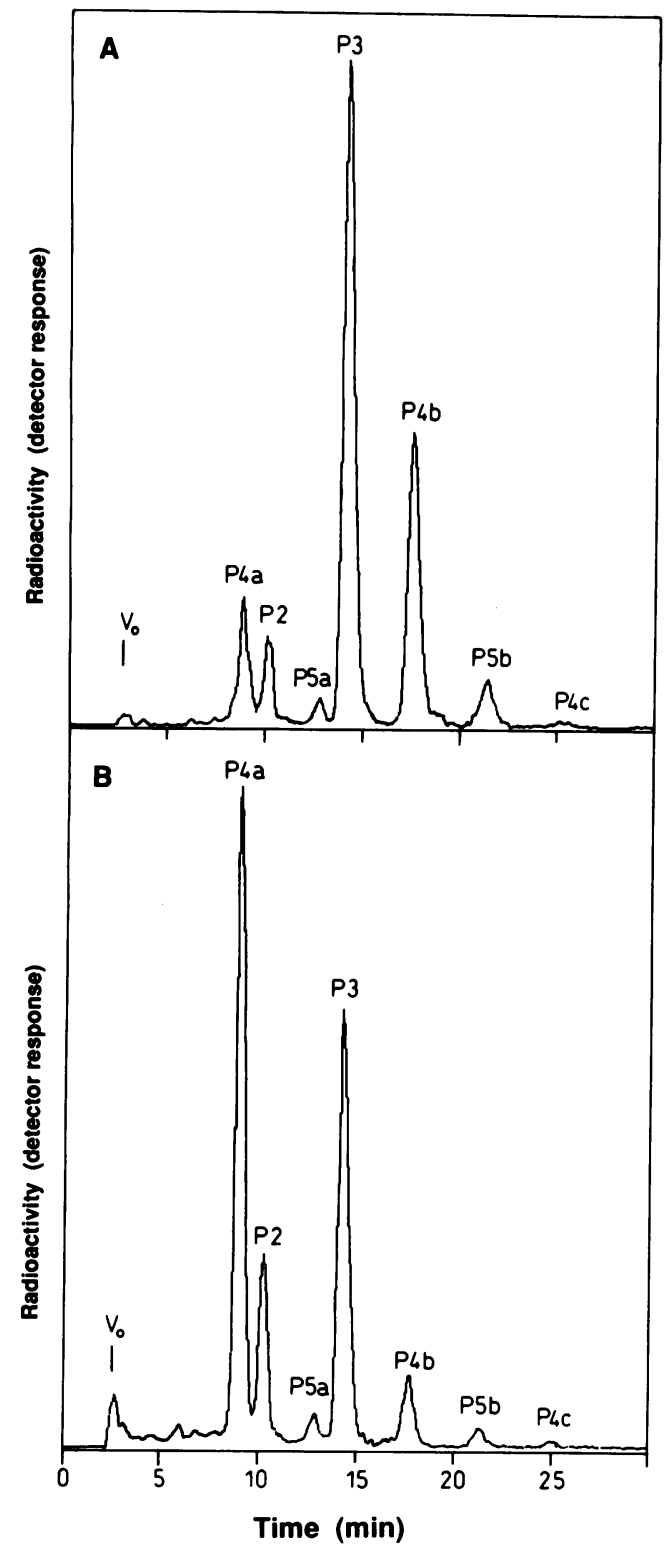

Fig. 2. HPLC of the phosphorylated oligosaccharide repeat units from P-LPG (panel A) and M-LPG (panel B). The LPG preparations were depolymerized by acid hydrolysis $(40 \mathrm{mM}$ trifluoroacetic acid, $8 \mathrm{~min}$, $100^{\circ} \mathrm{C}$ ) and the released phosphorylated oligosaccharides were labelled at the reducing terminus by $\mathrm{NaB}_{3} \mathrm{H}_{4}$ reduction. The labelled, phosphorylated oligosaccharides were analyzed by Dionex HPLC (program a) and identified by their coelution with unlabelled repeat units derived from L.major LPG and by chemical and enzymatic characterization (Table I). terminating side chains during metacyclogenesis, the actual number of repeat units containing these side chains per molecule is the same in both P- and M-LPG, raising the possibility that M-LPG could be formed by elongation of P-LPG with Ara-containing repeat units. Regardless of the mechanism of synthesis it is likely that the replacement of P-LPG with M-LPG will be facilitated by the rapid turnover of surface LPG which is continuously shed from the cell surface (Handman et al., 1984; King et al., 1987). This shedding appears to result from the passive partitioning of

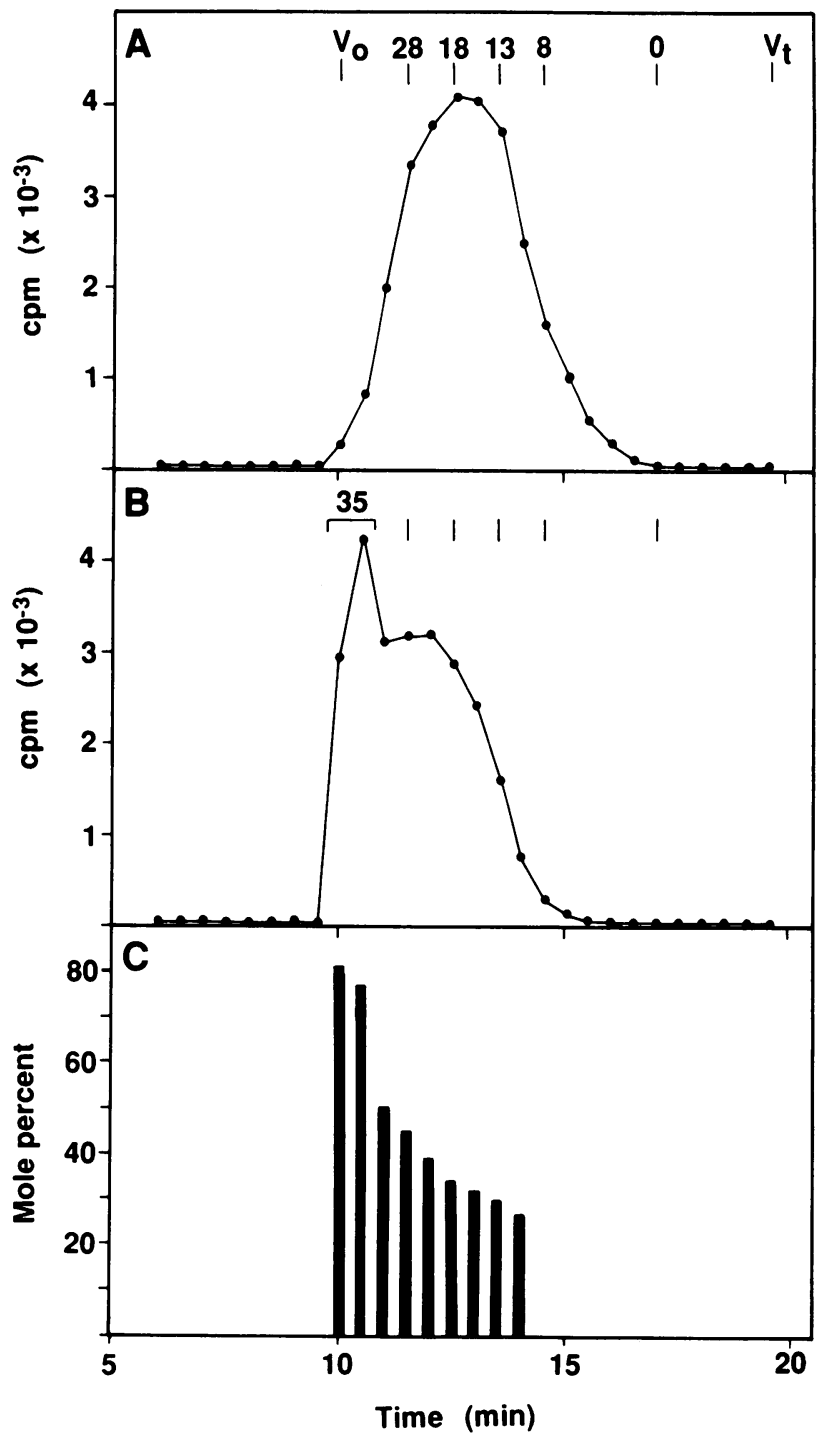

Fig. 3. HPLC gel permeation chromatography of P- and M-LPG. The LPG preparations were radiolabelled by treatment with galactose oxidase and $\mathrm{NaB}_{3} \mathrm{H}_{4}$, repurified by octyl-Sepharose chromatography and then delipidated with PI-specific phospholipase $\mathrm{C}$. The labelled phosphoglycans derived from P-LPG (panel A) and M-LPG (panel B) were chromatographed on a TSK2000SW gel permeation column. The numbers at the top of each profile refer to the average number of repeat units per molecule in each fraction. These values were estimated from the ratio of mannose to myo-inositol in each fraction when unlabelled phosphoglycan $(6 \mathrm{nmol})$ was coinjected with the labelled material. The elution position of the glycan core with no repeat units $(0)$ was determined using the glycan moiety from the truncated LPG produced by the LPG-deficient L.major strain LRCL1 19 (McConville and Homans, 1992). The increase in the relative abundance in the arabinosylated repeat units with increasing phosphoglycan chain length in metacyclic LPG is shown in panel $\mathbf{C}$. These values were calculated from the ratio of Ara to Man in each fraction and are expressed as mole percent. 
intact LPG molecules out of the plasma membrane as either monomers or micelles into the culture supernatant (Ilg et al., 1992). The high rate of shedding is probably due to the weak attachment of these molecules to the lipid bilayer of the plasma membrane via a single alkyl chain (McConville, 1991). It is of interest that some P-LPG is apparently retained by metacyclic promastigotes. This is suggested by the presence in the M-LPG pool of species which were

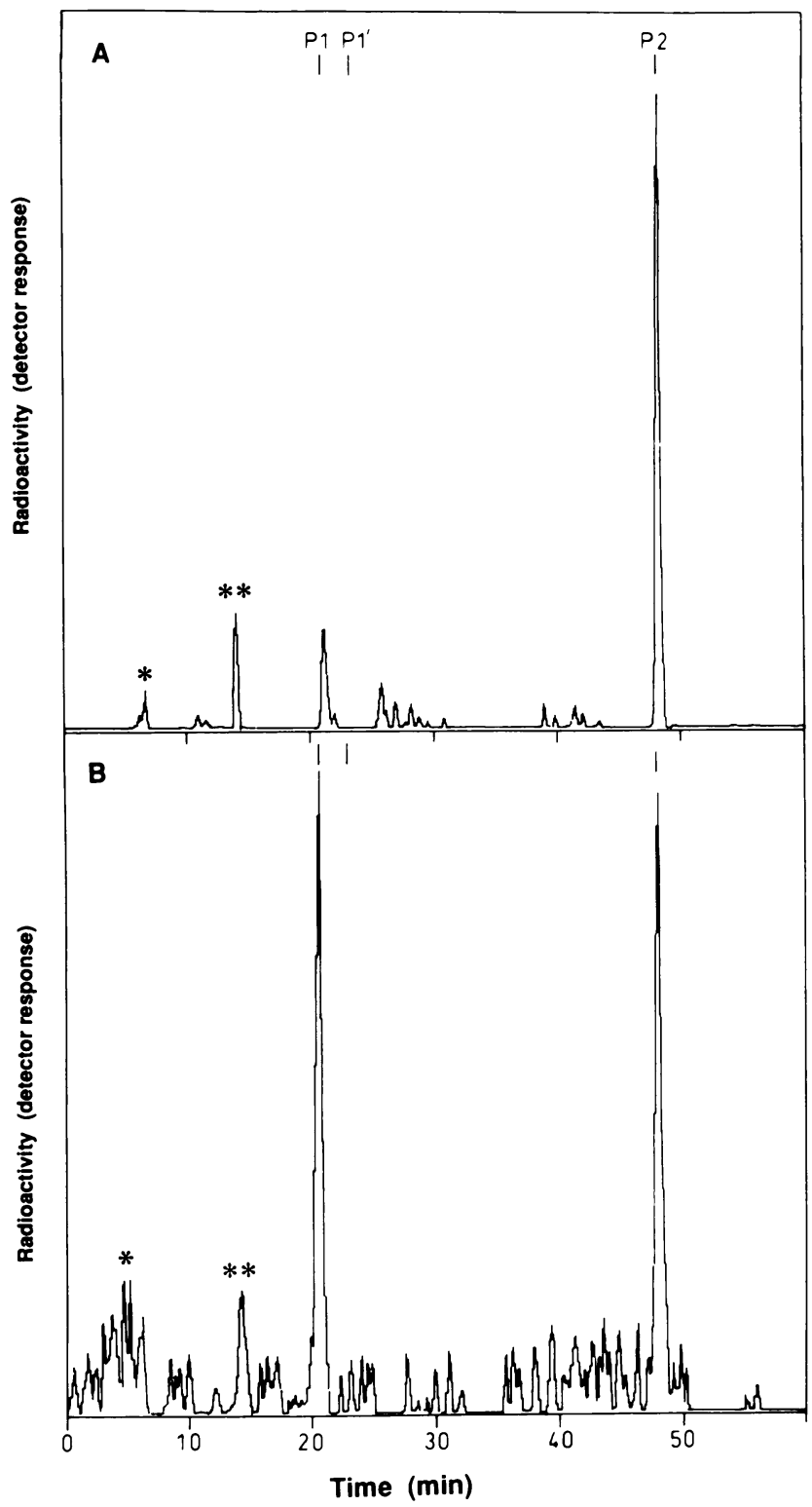

Fig. 4. HPLC analysis of the glycan cores of P and M-LPG. Following mild acid depolymerization, the GPI anchors of the LPGs were purified by octyl-Sepharose chromatography, delipidated with PIspecific phospholipase $\mathrm{C}$, then deaminated and radiolabelled by reduction with $\mathrm{NaB}_{3} \mathrm{H}_{4}$. The labelled glycan cores derived from PLPG (panel A) and M-LPG (panel B) were analyzed by Dionex HPLC using gradient program $\mathrm{b}$. The elution positions of the defined monophosphorylated glycans, $\mathrm{PO}_{4}-6 \mathrm{Gal} \alpha 1-6 \mathrm{Gal} \alpha 1-3 \mathrm{Gal} f \beta 1-3 \mathrm{Man} \alpha 1-3-$ Man $\alpha 1-4 \mathrm{AHM}(\mathrm{P} 1)$ and Gal $\alpha 1-6 \mathrm{Gal} \alpha 1-3 \mathrm{Gal} f \beta 1-3\left[\mathrm{PO}_{4}-6\right] \mathrm{Man} \alpha 1-3-$ Man $\alpha 1-4 \mathrm{AHM}$ ( $\left.\mathrm{Pl}^{\prime}\right)$ and the diphosphorylated glycan core. $\mathrm{PO}_{4}-6 \mathrm{Gal} \alpha$ 1-6Gal $\alpha 1-3 \mathrm{Gal} f \beta 1-3\left[\mathrm{PO}_{4}-6\right] \mathrm{Man} \alpha 1-3 \mathrm{Man} \alpha$ 1-4AHM (P2) derived from L.major LPG (McConville et al., 1990) are indicated. Peaks marked $*$ and $* *$ were derived from the mono- and diphosphorylated glycan cores, respectively, as a result of partial cleavage of the acid labile glycosidic linkage betwen the $\beta$-galactofuranose and mannose residues during nitrous acid deamination (McConville et al.. 1990a) indistinguishable from P-LPG in having a relatively low molecular weight and few Ara-containing repeat units (Figure 3C). These species are poorly labelled when metacyclic promastigotes are incubated with radioactive sugars (unpublished data), supporting the notion that they represent a pool of P-LPG which has either been sequestered within an intracellular compartment or retained on the surface membrane of metacyclic promastigotes.

The changes in the relative abundance of different repeat units may be functionally important in regulating the attachment of promastigotes to the midgut of the sandfly vector. It has been shown recently that LPG is involved in mediating attachment of actively dividing promastigotes to epithelial cells in the sandfly midgut (Pimenta et al., 1992). Binding can be inhibited with P-LPG or the purified repeat units, P3 or P4b, but not with M-LPG or the repeat unit, $\mathrm{P} 4 \mathrm{a}$, suggesting that the receptor on the epithelial cells is binding to the terminal $\beta \mathrm{Gal}$ residues on the LPG side chains. Thus the replacement of P-LPG with M-LPG during metacyclogenesis is likely to be one mechanism by which metacylic promastigotes detach from epithelial cells in the midgut, a process that is required for anterior migration and subsequent transmission to the mammalian host. It is of interest that M-LPG retains some Gal-terminating side chains. These side chains are still accessible to macromolecules as they can be oxidized with galactose oxidase in vitro and on the promastigote cell surface

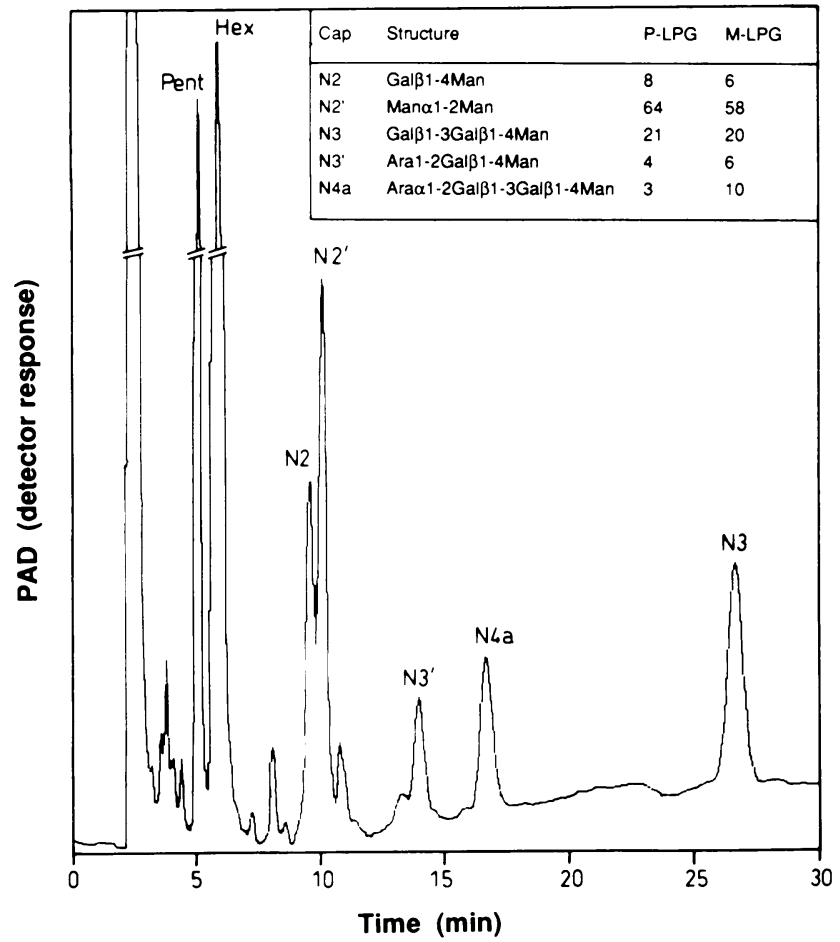

Fig. 5. HPLC analysis of the neutral cap species of M-LPG. The neutral oligosaccharides released from M-LPG by mild acid hydrolysis were purified as described in Materials and methods and analyzed by Dionex HPLC using program c. The structures of these species were deduced from their chromatographic coelution with authentic standards and by methylation analysis of the purified fractions (Table II). In addition to the cap structures, this fraction contained free

monosaccharides. which were shown by GC-MS analysis to be arabinose (Pent) or glucose and galactose (Hex). The presence of these monosaccharides reflects the release of some $(<5 \%)$ of the terminal monosaccharides from the repeat units and the hydrolysis of all the Glc residues that are in acid labile phosphodiester linkage to the glycan core (McConville and Homans. 1992: Thomas et al., 1992). 
(unpublished data). As M-LPG is ineffective at inhibiting attachment of procyclic promastigotes to the midgut wall (Pimenta et al., 1992) these data suggest that the receptor has a low affinity for $\beta \mathrm{Gal}$ side chains which are infrequently expressed along the phosphoglycan chain and that it may require clusters of these side chains for high affinity binding. This raises the possibility that secreted P-LPG, which is abundant within the lumen of the sandfly midgut (Davies et al., 1990), will enhance promastigote detachment or prevent reattachment to the midgut wall by effectively competing for midgut receptors.

Developmentally regulated changes in the nature of the LPG side chains may also influence the way in which promastigotes adhere to and subsequently invade macrophages. Several groups have shown that while procyclic forms can bind directly to macrophages in the absence of exogenous complement, metacyclic promastigotes, which are likely to be the main forms to initiate infection in the mammalian host, need to be opsonized with complement components before they are phagocytosed by macrophages via the complement receptors, CR1 or CR3 (da Silva et al., 1989; Mosser et al., 1992). There is evidence that the direct binding of procyclics to macrophages is mediated, at least in part, by LPG (Handman and Goding, 1984; Talamas-Rohana et al., 1990) and that the macrophage receptors preferentially recognize the Gal-terminating side chains of LPG with two or more residues (Kelleher et al., 1992). The down-regulation of these LPG epitopes during metacyclogenesis may account for the diminished ability of metacyclic promastigotes to bind directly to macrophage receptors and may encourage promastigote uptake via the complement receptors. Internalization via these receptors is thought to enhance promastigote survival within the macrophage as phagocytosis via these receptors is not coupled to the release of toxic oxygen metabolites (Wright and Silverstein, 1983).

LPG is the major activator of complement on the

Table II. Methylation analysis of the neutral cap oligosaccharides from M-LPG

\begin{tabular}{|c|c|c|c|c|c|c|}
\hline PMAA & Origin & $\mathrm{N} 2$ & $\mathrm{~N} 2^{\prime}$ & $\mathrm{N} 3$ & N3' & $\mathrm{N} 4 \mathrm{a}$ \\
\hline \multicolumn{7}{|l|}{ Arabinitol } \\
\hline 2,3,4-tri- $O$-methyl & Terminal Ara & 0.0 & 0.0 & 0.0 & 0.7 & 0.6 \\
\hline \multicolumn{7}{|l|}{ Galactitol } \\
\hline $2,3,4,6$-tetra- $O$-methyl & Terminal Gal & 0.9 & 0.0 & 1.3 & 0.0 & 0.0 \\
\hline 2,4,6-tri- $O$-methyl & 3-O-substituted Gal & 0.0 & 0.0 & 1.2 & 0.0 & 0.9 \\
\hline 3,4,6-tri- $O$-methyl & 2-O-substituted Gal & 0.0 & 0.0 & 0.0 & 1.2 & 0.7 \\
\hline \multicolumn{7}{|l|}{ Mannitol } \\
\hline 2,3,4,6-tetra- $O$-mthyl & Terminal Man & 0.0 & 0.8 & 0.0 & 0.0 & 0.0 \\
\hline 3,4,6-tri- $O$-methyl & 2-O-substituted Man & 0.0 & 1.0 & 0.0 & 0.0 & 0.0 \\
\hline 2,3,6-tri- $O$-methyl & 4- $O$-substituted Man & 1.0 & 0.0 & 1.0 & 1.0 & 1.0 \\
\hline
\end{tabular}

The permethylated alditol acetates (PMAAs) were identified and quantified by GC-MS using empirically determined response factors.
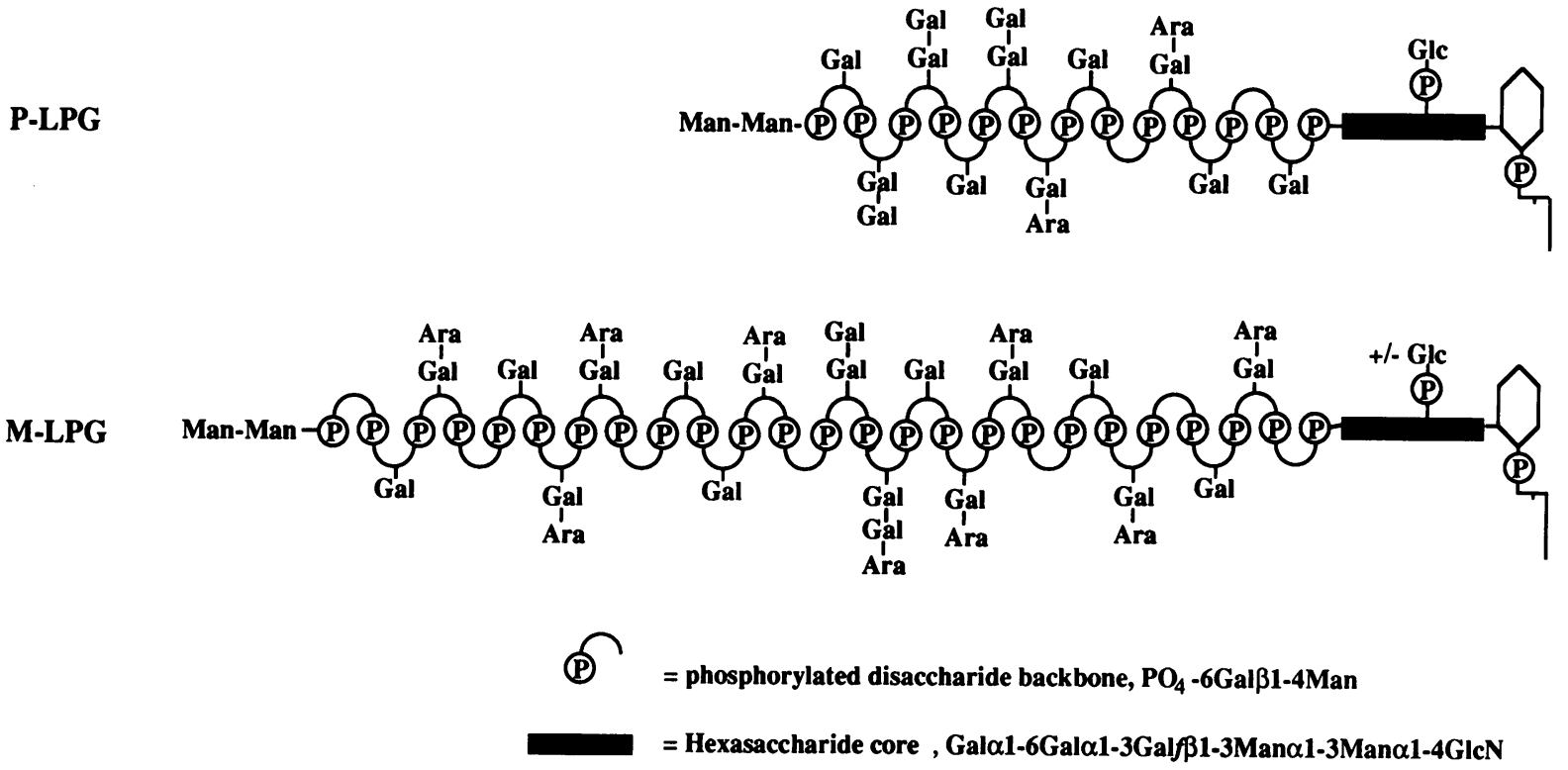

Fig. 6. Schematic diagram of the structures of the LPG from procyclic and metacyclic L.major promastigotes. The major developmental modifications during metacyclogenesis include an approximate doubling in the average number of repeat units per molecule, a dramatic increase in the number of repeat units with side chains of Arap $\alpha 1-2 \mathrm{Gal} \beta 1-3$ and a decrease in the extent to which the glycan core is substituted with $\mathrm{Glc} \alpha 1-\mathrm{PO}_{4}$. Both structures contain the same hexasaccharide glycan core and the disaccharide, Man $\alpha 1-2 \mathrm{Man}$, as the principle cap structure. It is not known whether the different repeat units are randomly distributed along the phosphoglycan chain, as shown here, or whether some repeat units are preferentially localized at one end of the chain. 
promastigote surface, leading to the deposition of both $\mathrm{C} 3 \mathrm{~b}$ and the lytic complex C5b-9 on both developmental stages (Puentes et al., 1988, 1990). It has been suggested that the longer LPG chains on the surface of metacylic promastigotes may increase the resistance of this stage to complementmediated lysis by preventing the insertion of the C5b-9 complex into the plasma membrane (Sacks et al., 1990). This is supported by the observation that while $\mathrm{C} 5 \mathrm{~b}-9$ binds tightly to procyclic promastigotes, resulting in cell lysis, it is rapidly shed from the surface of metacyclic promastigotes which are correspondingly more resistant to complementmediated lysis (Puentes et al., 1990). The approximate doubling in the length of the LPG chains may prevent $\mathrm{C} 5 \mathrm{~b}-9$ insertion into the parasite membrane by initially activating complement further away from the plasma membrane, and then sterically hindering access of this complex to the membrane. This resistance to complement-mediated lysis appears to be one of the key factors contributing to the increased infectivity of metacyclic promastigotes.

\section{Materials and methods}

\section{Strains}

L.major promastigotes of strain V121 (derived by limiting dilution from the L.major strain LRC L37) or the Friendlin strain, clone VI, were cultivated in Schneider's Drosophila medium supplemented with $10 \%$ fetal calf serum or Grace's insect medium supplemented with $20 \%$ fetal calf serum, respectively. Metacyclic promastigotes were obtained from cultures in stationary growth phase (5-6 day old) after agglutination of non-metacyclic promastigotes with $50 \mu \mathrm{g} / \mathrm{ml}$ peanut agglutinin as previously described (Sacks and da Silva, 1987).

\section{Purification of $L P G$}

LPG was extracted from delipidated cells with 9\% 1-butanol and purified by two rounds of octyl-Sepharose chromatography (McConville et al., 1987, 1990a). In some experiments, LPG was purified by an alternative procedure involving solvent extraction and gel filtration (Sacks et al., 1990).

\section{Depolymerization of LPG}

The purified LPGs were depolymerized in $40 \mathrm{mM}$ trifluoroacetic acid $\left(100^{\circ} \mathrm{C}, 8 \mathrm{~min}\right)$. The acid was removed under reduced pressure and the hydrolysate was resuspended in $0.1 \mathrm{M} \mathrm{NH}_{4} \mathrm{OAc}$ containing 5\% 1-propanol and loaded onto a column (1 ml) of octyl-Sepharose equilibrated in the same buffer. The phosphorylated oligosaccharide repeat units and neutral cap structures were eluted with four $0.5 \mathrm{ml}$ aliquots of $0.1 \mathrm{M} \mathrm{NH}_{4} \mathrm{OAc} / 5 \%$ 1-propanol, while the GPI glycolipid anchor was eluted from the column with 35\% 1-propanol. Carbohydrate-containing fractions were detected by spotting 1-2 $\mu \mathrm{l}$ aliquots of each fraction onto aluminium-backed HPTLC silica gel sheets (Merck) and staining with orcinol- $-\mathrm{H}_{2} \mathrm{SO}_{4}$. After lyophilization to remove volatile buffer salts, these fractions were analyzed as described below.

\section{Analysis of the repeat units}

The phosphorylated oligosaccharide repeat units were reduced with $\mathrm{NaB}_{3} \mathrm{H}_{4}$ $(12 \mathrm{mM})$ in $20 \mu \mathrm{l} 0.1 \mathrm{M} \mathrm{NaOH}$-boric acid buffer, $\mathrm{pH} 10\left(25^{\circ} \mathrm{C}, 2 \mathrm{~h}\right)$. The mixture was acidified with $1 \mathrm{M}$ acetic acid and desalted by passage down a column of AG50X12( $\left.\mathrm{H}^{+}\right)$, followed by methanol evaporation. The radiolabelled glycans were purified from radiochemical impurities by descending paper chromatography on Whatman $3 \mathrm{MM}$ paper in 1-butanol/ethanol/water $(4: 1: 0.6 \mathrm{v} / \mathrm{v} / \mathrm{v})$. The ${ }^{3} \mathrm{H}$-labelled glycans were analyzed by HPLC on a Dionex model BioLC Carbohydrate Analyser (Dionex Corporation, Sunnyvale, CA) equipped with a pulsed amperometric detector and a Raytest Ramona radioactivity flow monitor. All the monophosphorylated repeat units were resolved on a CarboPac PAl column $(4 \times 250 \mathrm{~mm})$ which was eluted with a linear gradient of $\mathrm{NaOAc}(0.175-0.25$ M) in $0.15 \mathrm{M} \mathrm{NaOH}$ over $40 \mathrm{~min}$ at a flow rate of $0.6 \mathrm{ml} / \mathrm{min}$ (program a). Fractions containing labelled repeat units were desalted by passage down a $0.5 \mathrm{ml}$ column of AG50X12( $\left.\mathrm{H}^{+}\right)$, and acetic acid was removed by flash evaporation of the dried material with toluene. Methylation analysis and enzyme digestions of the dephosphorylated repeat units were performed as described previously (McConville et al., 1990a; McConville and Blackwell, 1991).

\section{Analysis of the glycan core}

The acid-released glycolipid anchor was delipidated with Bacillus thuringiensis PI-specific phospholipase $\mathrm{C}$ in $20 \mathrm{mM}$ Tris-acetate buffer, $\mathrm{pH} 7.5$, containing $0.1 \%$ Triton $\mathrm{X}-100\left(5 \mu \mathrm{l}, 37^{\circ} \mathrm{C}, 1 \mathrm{~h}\right)$ and then deaminated by addition of $0.25 \mathrm{M} \mathrm{NaNO}_{2}$ in $0.3 \mathrm{M} \mathrm{NaOAc}$ buffer, $\mathrm{pH} 4.0(20 \mu \mathrm{l}$, $40^{\circ} \mathrm{C}, 2 \mathrm{~h}$ ). The mixture was adjusted to $\mathrm{pH} 9$ with $400 \mathrm{mM}$ boric acid $(5 \mu \mathrm{l})$ and $1 \mathrm{M} \mathrm{NaOH}(8 \mu \mathrm{l})$ prior to the addition of $30 \mathrm{mM} \mathrm{NaB}^{3} \mathrm{H}_{4}$ in $0.1 \mathrm{M} \mathrm{NaOH}(5 \mu \mathrm{l})$ to reduce the newly formed terminal 2,5-anhydromannose residue. After $2 \mathrm{~h}$ incubation at $25^{\circ} \mathrm{C}$, excess reductant was destroyed with $1 \mathrm{M}$ acetic acid $(5 \mu \mathrm{l})$ and the mixture was desalted and subjected to descending paper chromatography as described above. The labelled phosphorylated glycan cores were analyzed by Dionex HPLC on a CarboPac PA1 column which was eluted with two linear gradients, from 175 to $250 \mathrm{mM} \mathrm{NaOAc}$ in $100 \mathrm{mM} \mathrm{NaOH}$ over $30 \mathrm{~min}$ and then from 250 to $500 \mathrm{mM} \mathrm{NaOAc}$ in $100 \mathrm{mM} \mathrm{NaOH}$ over $20 \mathrm{~min}$, at a flow rate of $0.6 \mathrm{ml} / \mathrm{min}$ (program b).

\section{Analysis of the cap oligosaccharides}

The neutral cap oligosaccharides were separated from the phosphorylated oligosaccharide repeat units by passage down a tandem column of AG50X12 $\left(\mathrm{H}^{+}\right)(0.3 \mathrm{ml})$ over AG3X4(OH $\left.{ }^{-}\right)(0.3 \mathrm{ml})$. The cap oligosaccharides were analyzed by Dionex HPLC on a CarboPac PA1 column which was eluted with a linear gradient of $\mathrm{NaOAc}(12-72 \mathrm{mM}$, $80 \mathrm{~min}$ ) in $100 \mathrm{mM} \mathrm{NaOH}$ at a flow rate of $0.6 \mathrm{ml} / \mathrm{min}$ (program c).

\section{HPLC gel permeation chromatography}

LPG was labelled by the galactose oxidase $/ \mathrm{NaB}^{3} \mathrm{H}_{4}$ procedure. LPG was treated with galactose oxidase ( 2 units) in $0.1 \mathrm{M}$ sodium phosphate buffer, $\mathrm{pH} 7.0\left(37^{\circ} \mathrm{C}, 2 \mathrm{~h}\right)$. The $\mathrm{pH}$ was then adjusted to 9 by addition of 400 $\mathrm{mM}$ boric acid $(5 \mu \mathrm{l})$ and $1 \mathrm{M} \mathrm{NaOH}(3 \mu \mathrm{l})$ and the oxidized galactose residues were reduced with $30 \mathrm{mM} \mathrm{NaB}^{3} \mathrm{H}_{4}(5 \mu \mathrm{l})$ in $0.1 \mathrm{M} \mathrm{NaOH}$ for $1 \mathrm{~h}$ at $25^{\circ} \mathrm{C}$. The $\mathrm{pH}$ was adjusted to 5 with $1 \mathrm{M}$ acetic acid, prior to diluting the mixture with 4 vol of $0.1 \mathrm{M} \mathrm{NH}_{4} \mathrm{OAc}$ containing 5\% 1-propanol and loading it onto a column (1 ml) of octyl-Sepharose equilibrated in the same buffer. The column was washed with $10 \mathrm{ml}$ of $0.1 \mathrm{M} \mathrm{NH}_{4} \mathrm{OAc}$ containing 1-propanol prior to eluting the LPG with $1 \mathrm{ml}$ of $35 \%$ 1-propanol. After removal of buffer salts by lyophilization the ${ }^{3} \mathrm{H}$-labelled LPG was delipidated with Bacillus thuringiensis PI-specific phospholipase $\mathrm{C}$ and chromatographed on a TSK2000SW column $(25 \times 400 \mathrm{~mm})$ in $0.2 \mathrm{M}$ $\mathrm{NH}_{4} \mathrm{OAc}$ at a flow rate of $0.6 \mathrm{ml} / \mathrm{min}$. In order to calibrate the column, unlabelled phosphoglycan, derived from the LPG of stationary phase promastigotes, was coinjected with the labelled phosphoglycan and individual fractions were analyzed for inositol and mannose to determine the average number of repeat units per phosphoglycan chain in each size fraction.

\section{Monosaccharide and lipid analyses}

The neutral monosaccharides and 1-O-alkylglycerols were quantified by GC-MS as their trimethylsilyl derivatives after solvolysis in $0.5 \mathrm{M}$ methanolic $\mathrm{HCl}\left(50 \mu \mathrm{l}, 80^{\circ} \mathrm{C}, 16 \mathrm{~h}\right)$, as previously described (Chaplin, 1982; McConville and Bacic, 1989).

Response factors for determining the ratio of mannose to 1-O-alkylglycerol in LPG were derived from authentic glycolipid species purified from $L$ major which contained the same alkylglycerol species (McConville et al., 1990b). The myo-inositol content was measured by GC-MS after acid hydrolysis $\left(6 \mathrm{M} \mathrm{HCl}, 110^{\circ} \mathrm{C}, 16 \mathrm{~h}\right.$ ) and trimethylsilyl derivatization (McConville et al., 1990a).

\section{Acknowledgments}

We would like to thank Dr M.Low for generously giving the PI-specific phospholipase $C$. This work was supported by the Wellcome Trust and the Medical Research Council. M.J.M. is the recipient of a Wellcome Trust Senior Research Fellowship. S.J.T. is a Burroughs Wellcome Scholar in Molecular Parasitology.

\section{References}

Chaplin,M.F. (1982) Anal. Biochem., 123, 336-341.

da Silva,R.P., Hall,B.F., Joiner,K.A. and Sacks,D.L. (1989) J. Immunol., 143, 617-622.

Davies,C.R., Cooper,A.M., Peacock,C., Lane,R.P. and Blackwell,J.M. (1990) Parasitology, 101, 337-343.

Elhay,M.J., Kelleher,M., Bacic,A., McConville,M.J., Tolson,D.L., Pearson,T.W. and Handman,E. (1990) Mol. Biochem. Parasitol., 40, $151-300$

Handman,E. and Goding,J.W. (1985) EMBO J., 4, 329-336. 
Handman,E.H., Greenblatt,C.L. and Goding,J.W. (1984) EMBO J., 3, $2301-2306$

Homans,S.W., Melhert,A. and Turco,S.J. (1992) Biochemistry, 31, $654-661$.

Ilg,T., Etges,R., Overath,P., McConville,M.J., Thomas-Oates,J., Thomas,J., Homans,S.W. and Ferguson,M.A.J. (1992) J. Biol. Chem., 267, 6834-6840.

Kelleher,M., Bacic,A. and Handman,E. (1992) Proc. Natl. Acad. Sci. USA, 89, 6-10.

King,D.L., Chang,Y.D. and Turco,S.J. (1987) Mol. Biochem. Parasitol., 24, 47-53.

McConville,M.J. (1991) Cell Biol. Int. Rep., 15, 779-798.

McConville,M.J. and Bacic,A. (1989) J. Biol. Chem., 264, 757-766.

McConville,M.J. and Bacic,A. (1990) Mol. Biochem. Parasitol., 38, 57-68.

McConville,M.J. and Blackwell,J.M. (1991) J. Biol. Chem., 266, 15170-15179.

McConville,M.J. and Homans,S.W. (1992) J. Biol. Chem., 267, $5855-5861$.

McConville,M.J., Bacic,A., Mitchell,G.F. and Handman,E. (1987) Proc. Natl. Acad. Sci. USA, 84, 8941-8945.

McConville,M.J., Thomas-Oates,J.E., Ferguson,M.A.J. and Homans,S.W. (1990a) J. Biol. Chem., 265, 19611-19623.

McConville,M.J., Homans,S.W., Thomas-Oates,J.E., Dell,A. and Bacic,A. (1990b) J. Biol. Chem., 265, 7385-7394 .

McNeely,T.B. and Turco,S.J. (1990) J. Immunol., 144, 2745-2750.

Mosser,D.M., Springer,T.A. and Diamond,M.S. (1992) J. Cell Biol., 116, $511-520$.

Pimenta,P.F., da Silva,R.P., Sacks,D.L. and da Silva,P.P. (1989) Eur. J. Cell Biol., 48, 180-190.

Pimenta,P.F.P., Turco,S.J., McConville,M.J., Lawyer,P.G., Perkins,P.V. and Sacks,D.L. (1992) Science, 256, 1812-1815.

Puentes,S.M., Sacks,D.L., da Silva,R.P. and Joiner,K.A. (1988) J. Exp. Med., 167, 887-902.

Puentes,S.M., da Silva,R.P., Sacks,D.L., Hammer,C.H. and Joiner,K.A. (1990) J. Immunol., 145, 4311-4316.

Sacks,D.L. (1989) Exp. Parasitol., 69, 100-103.

Sacks,D.L. and da Silva,R.P. (1987) J. Immunol., 139, 3099-3106.

Sacks,D.L. and Perkins,P. (1984) Science, 223, 1417-1419.

Sacks,D.L., Brodin,T.N. and Turco,S.J. (1990) Mol. Biochem. Parasitol., 42, 225-234.

Talamas-Rohana,P., Wright,S.D., Lennartz,M.R. and Russell,D.G. (1990) J. Immunol., 144, 4817-4824.

Thomas,J.R., McConville,M.J., Thomas-Oates,J.E., Homans,S.W., Ferguson,M.A.J., Gorin,P.A.J, Greis,K.D. and Turco,S.J. (1992) J. Biol. Chem., 267, 6829-6833.

Turco,S.J. (1989) Exp. Parasitol., 70, 241-245.

Turco,S.J., Orlandi,P.A.,Jr, Homans,S.W., Ferguson,M.A.J., Dwek,R.A. and Rademacher,T.W. (1989) J. Biol. Chem., 264, 6711-6715.

Wright,S.D. and Silverstein,S.C. (1983) J. Exp. Med., 158, 2016-2023.

Received on May 7, 1992; revised on June 24, 1992 